\title{
Random Dynamics of the Stochastic Boussinesq Equations Driven by Lévy Noises
}

\author{
Jianhua Huang, 1 Yuhong Li, ${ }^{2}$ and Jinqiao Duan ${ }^{3}$ \\ ${ }^{1}$ Department of Mathematics, National University of Defense Technology, Changsha 410073, China \\ ${ }^{2}$ College of Hydropower and Information Engineering, Huazhong University of Science and Technology, Wuhan 430074, China \\ ${ }^{3}$ Department of Applied Mathematics, Illinois Institute of Technology, Chicago, IL 60616, USA
}

Correspondence should be addressed to Jianhua Huang; jhhuang32@nudt.edu.cn

Received 13 December 2012; Accepted 17 January 2013

Academic Editor: Chuangxia Huang

Copyright (C) 2013 Jianhua Huang et al. This is an open access article distributed under the Creative Commons Attribution License, which permits unrestricted use, distribution, and reproduction in any medium, provided the original work is properly cited.

\begin{abstract}
This paper is devoted to the investigation of random dynamics of the stochastic Boussinesq equations driven by Lévy noise. Some fundamental properties of a subordinator Lévy process and the stochastic integral with respect to a Lévy process are discussed, and then the existence, uniqueness, regularity, and the random dynamical system generated by the stochastic Boussinesq equations are established. Finally, some discussions on the global weak solution of the stochastic Boussinesq equations driven by general Lévy noise are also presented.
\end{abstract}

\section{Introduction}

Dynamical systems driven by non-Gaussian processes, such as Lévy processes, have attracted a lot of attention recently. Ordinary differential equations driven by Lévy processes have been summarized in [1]. Peszat and Zabczyk [2] have presented a basic framework for partial differential equations driven by Lévy processes.

The Navier-Stokes fluid equations are often coupled with other equations, especially, with the scalar transport equations for fluid density, salinity, or temperature. These coupled equations model a variety of phenomena arising in environmental, geophysical, and climate systems. The related Boussinesq fluid equations [3-5] under Gaussian fluctuations have been recently studied, for example, existence and uniqueness of solutions [6], stochastic flow, dynamical impact under random dynamical boundary conditions $[7,8]$, and large deviation principles $[9,10]$, among others.

Motivated by a recent work on a simple stochastic partial differential equation with Lévy noise [11], we study the stochastic Boussinesq equations driven by some special Lévy noises, and we consider the random dynamics of this stochastic system. Specifically, for a given bounded $C^{1}$ smooth domain $D \subset \mathbb{R}^{2}$ with sufficient smooth boundary, we consider the following stochastic Boussinesq equations driven by subordinator Lévy noise:

$$
\begin{gathered}
\frac{d u}{d t}=\left(\frac{1}{\operatorname{Re}} \Delta u-\nabla p-u \cdot \nabla u-\frac{1}{\mathrm{Fr}^{2}} \theta e_{2}\right)+d Y_{1}(t), \\
\text { on } D \times \mathbb{R}_{+}, \\
\frac{d \theta}{d t}=\left(\frac{1}{\operatorname{RePr}} \Delta \theta-u \cdot \nabla \theta\right)+d Y_{2}(t), \quad \text { on } D \times \mathbb{R}_{+}, \\
\operatorname{div} u=0, \quad \text { on } D \times \mathbb{R}_{+}, \\
u(0)=u_{0}, \quad \theta(0)=\theta_{0},
\end{gathered}
$$

where $u=u(x, t)=\left(u^{1}, u^{2}\right) \in \mathbb{R}^{2}$ is the velocity vector, $\theta=\theta(t, x) \in \mathbb{R}$ is salinity, $p(t, x) \in \mathbb{R}$ is the pressure term, $x=(\xi, \eta) \in D \subset \mathbb{R}^{2}, \Delta$ denotes the Laplacian operator, and $\nabla$ denotes the gradient operator. Moreover, Fr is the Froude number, Re is the Reynolds number, $\mathrm{Pr}$ is the Prandtl number, and $e_{2} \in \mathbb{R}^{2}$ is a unit vector in the upward vertical direction. The initial data $u_{0}, \theta_{0}$ are given. Both $Y_{1}(t)$ and $Y_{2}(t)$ are subordinator Lévy processes on Hilbert spaces $H_{1}$ and $H_{2}$, which will be specified later. The present paper is 
devoted to the existence, uniqueness, regularity, and the cocycle property of solution for stochastic Boussinesq equations (1).

This paper is organized as follows. In Section 2, we first present some properties of the subordinator Lévy process $Y(t)$, then review some fundamental properties of the stochastic integral with respect to Lévy process $Y(t)$. Section 3 is devoted to the existence, uniqueness, regularity, and the cocycle property of the stochastic Boussinesq equations. Finally, some discussions on the global weak solution of stochastic Boussinesq equations driven by general Lévy noise are also presented in Section 4.

\section{Preliminaries}

In this section, we introduce some operators and fraction spaces and then present some properties of the subordinator Lévy process $Y(t)$ and the stochastic integral with respect to Lévy process $Y(t)$.

In order to reformulate the stochastic Boussinesq equations (1) as an abstract stochastic evolution, we introduce the following function spaces.

Denote $L^{2}(D)$ to be the space of functions defined on $D$, which are $L^{2}$-integrable with respect to the Lebesgue measure $d x=d x_{1} d x_{2}$, endowed with the usual scalar product and norm, that is, for $u, v \in L^{2}(D)$,

$$
(u, v)=\int_{D} u(x) v(x) d x, \quad|u|=\{(u, u)\}^{1 / 2} .
$$

For $m \in \mathbb{Z}^{+} \cup\{0\}$ and $q \in(1, \infty)$, define

$$
\begin{aligned}
& H^{m, q}(D) \\
& \quad=\left\{u \in L^{q}(D): D^{\alpha} u \in L^{q}(D), \alpha \in \mathbb{N}^{2}, 0 \leq|\alpha| \leq m\right\}
\end{aligned}
$$

as the usual Soblev space with scalar product

$$
(u, v)_{m}=\sum_{0 \leq|\alpha| \leq m}\left(D^{\alpha} u, D^{\alpha} v\right)_{L^{q}(D)}
$$

and the induced norm

$$
|u|_{m}=\|u\|_{H^{m}(D)}=\left(\sum_{0 \leq|\alpha| \leq m}\left|D^{\alpha} u\right|_{L^{q}(D)}^{q}\right)^{1 / 2},
$$

where $D^{\alpha} u$ is the $\alpha$ th order weak derivative of $u$.

For $s \in \mathbb{R}$, let $H^{s, q}(D)$ be defined by the complex interpolation method [12] as follows.

$$
H^{\beta, q}(D)=\left[H^{k, q}(D), H^{m, q}(D)\right]_{\theta}
$$

where $k, m \in \mathbb{N}, \theta \in(0,1)$, and $k<m$ are chosen to satisfy

$$
\beta=(1-\theta) k+\theta m
$$

The closure of $C_{0}^{\infty}(D)$ in the Banach space $H^{s, q}(D), s \geq 0$, $q \in(1, \infty)$, will be denoted by $H_{0}^{s, q}(D)$.
The following product spaces are needed:

$$
\begin{gathered}
\mathscr{V}=\left\{u=\left(u^{1}, u^{2}\right) \in\left(C^{\infty}(D)\right)^{2} \times C^{\infty}(D), \nabla \cdot u=0\right\}, \\
\mathbb{Q}^{q}(D)=\left(L^{q}(D)\right)^{2} \times L^{q}(D), \\
=\left\{u=\left(u^{1}, u^{2}\right) \in\left(H^{s, q}(D)\right)^{2} \times H^{s, q}(D),\right. \\
\nabla \cdot u=0\}, \\
\mathbb{V}^{s, q}(D)=\left(H^{s, q}(D)\right)^{2} \times H^{s, q}(D) \\
\left.\nabla u=\left(u^{1}, u^{2}\right) \in \mathbb{T}_{0}^{s, q}(D), \nabla \cdot u=0\right\} .
\end{gathered}
$$

Let $\mathbf{H}^{s, q}(D)$ denote the closure of $\mathscr{V}$ with respect to the $\dddot{H}^{s, q}$-norm, $V^{s, q}(D)$ denote the closure of $\mathscr{V}$ with respect to the $\mathbb{V}^{s, q}$-norm, and $V^{\prime}$ be the dual space of $V^{s, q}(D)$. In particular, we denote by $\mathbf{H}^{1,2}$ and $V^{1,2} H$ and $V$, respectively.

Denote

$$
\begin{gathered}
A_{1} u=\Delta u(t), \quad A_{2} \theta=\Delta \theta(t), \\
B_{1}\left(u_{1}, u_{2}\right)=\left(u_{1} \cdot \nabla\right) u_{2}, \quad B_{2}\left(u_{1}, \theta_{2}\right)=\left(u_{1} \cdot \nabla\right) \theta_{2}, \\
U_{0}=\left(\begin{array}{c}
u_{0} \\
\theta_{0}
\end{array}\right) \in H, \quad U(t)=\left(\begin{array}{l}
u(t) \\
\theta(t)
\end{array}\right) \in V, \\
R(U)=\left(\begin{array}{c}
-\frac{1}{\mathrm{Fr}^{2}} \theta e_{2}, \\
0
\end{array}\right) \\
Y(t)=\left(\begin{array}{c}
Y_{1}(t) \\
Y_{2}(t)
\end{array}\right) \in H=H_{1} \times H_{2}
\end{gathered}
$$

where $v=1 / \operatorname{Re}$ and $k=1 / \operatorname{Re} \operatorname{Pr}$.

Now, we define the following two operators:

$$
\begin{aligned}
& A: V \longrightarrow V^{\prime}: V \ni U=(u, \theta) \longmapsto A U=\left(\begin{array}{l}
v A_{1} u \\
k A_{2} \theta
\end{array}\right), \\
& B: V \times V \longrightarrow V^{\prime}: V \times V \ni\left(U_{1}, U_{2}\right) \\
& \longmapsto B\left(U_{1}, U_{2}\right)=\left(\begin{array}{l}
B_{1}\left(u_{1}, u_{2}\right) \\
B_{2}\left(u_{1}, \theta_{2}\right)
\end{array}\right) .
\end{aligned}
$$

Then, the stochastic Boussinesq system (1) can be rewritten as the following abstract stochastic evolution equation:

$$
\begin{gathered}
d U(t)+[A U(t)+B(U(t), U(t))+R(U(t))] d t=d Y(t), \\
U(0)=U_{0} .
\end{gathered}
$$

In order to apply the technique of the reproducing Kernel Hilbert space, it is better to introduce the definition $\gamma$ radonifying.

Definition 1 (see [13]). Let $K$ and $X$ be Banach spaces, a bounded linear operator $L: K \rightarrow X$ is called $\gamma$-radonifying 
if and only if $L\left(\gamma_{K}\right)$ is $\sigma$-additive, where $\gamma_{K}$ is the canonical cylindrical finitely additive set-valued function (also called a Gaussian distribution) on $K$.

The following is our standing assumption:

Assumption 1. Space $E \subset H \cap \mathbb{L}^{4}$ is a Hilbert space such that for some $\delta \in(0,1 / 2)$,

$$
A^{-\delta}: E \longrightarrow H \cap \mathbb{L}^{4} \text { is } \gamma \text {-radonifying. }
$$

Remark 2. Under the above assumption, we have the facts that $E \subset H$ and the Banach space $U$ is taken as $H \cap \mathbb{L}^{4}$ (see $[11,14,15]$ for more details and related results). In fact, space $E$ is the reproducing kernel Hilbert space of noise $W(t)$ on $H \cap \mathbb{L}^{4}$.

It is well known that subordinators form the subclass of increasing Lévy processes, which can be thought of as a random model of time evolution (see [16]). We will present some properties of the subordinator Lévy process $Y(t), t \geq 0$, then review briefly the stochastic integral with respect to Lévy process $Y(t)$.

Definition 3 (see $[1,2,11]$ ). Let $E$ be a Banach space, and let $Y=(Y(t), t \geq 0)$ be an $E$-valued stochastic process defined on a probability space $(\Omega, \mathscr{F}, \mathbb{P})$. Stochastic process $Y$ is called a Lévy process if

(L1) $Y(0)=0$, a.s.;

(L2) process $Y$ has independent and stationary increments; and

(L3) process $Y$ is stochastically continuous, that is, for all $\delta>0$ and for all $s \geq 0$,

$$
\lim _{t \rightarrow s} \mathbb{P}(|Y(t)-Y(s)|>\delta)=0 .
$$

A subordinator Lévy process is an increasing onedimensional Lévy process.

For $p>0, \operatorname{Sub}(p)$ denotes the set of all subordinator Lévy processes $Z$, whose intensity measure $\rho$ satisfies the condition $\int_{0}^{1} \eta^{p / 2} \rho(d \eta)<\infty$

In the most interesting cases, the space $E$ is a subspace of $H$, that is, $E \subset H$, and

$$
Y(t)=W(Z(t)), \quad t \geq 0,
$$

where $Z=(Z(t))_{t \geq 0}$ is an independent subordinator process belonging to class $\operatorname{Sub}(p), p \in(1,2], W=(W(t))_{t \geq 0}$ is an $H$ valued cylindrical Wiener process defined on some Banach space $U$.

We decompose the $H$-valued Lévy process $Y(t)$ into two parts $N_{1}(t)$ and $N_{2}(t)$, the first one with small jumps and the second one with (relatively) large jumps, that is,

$$
Y(t)=N_{1}(t)+N_{2}(t), \quad t \geq 0
$$

with $v$ being the intensity measure of Lévy process $Y, N_{1}$ being the Lévy process with the intensity measure:

$$
\begin{array}{r}
v_{1}(\Gamma)=v\left(\Gamma \cap B_{U}(0,1)\right), \quad \Gamma \in \mathscr{B}(U), \\
B_{U}(0,1) \text { denotes the unit ball in } U,
\end{array}
$$

and $N_{2}$ be the Lévy process with the intensity measure $v_{2}=$ $v-v_{1}$. Then $N_{2}$ can be defined as a compound Poisson process with the intensity measure $v_{2}$, and $N_{1}, N_{2}$ can be defined by the Poisson random measure $\pi$ which is defined as follows:

$$
\pi([0,1] \times \Gamma)=\sum_{s \leq t} 1_{\Gamma} \Delta Y(s), \quad \Gamma \in \mathscr{B}(U),
$$

where $\Delta Y(s)=Y\left(s^{+}\right)-Y\left(s^{-}\right), s \geq 0$. Here, the symbol $\Delta$ denotes the increment of $Y$.

We assume that the process $Y$ is right-continuous with left-hand side limits. Thus

$$
\Delta Y(s)=Y(s)-Y\left(s^{-}\right), \quad s \geq 0 .
$$

Notice that as $\pi$ is a time homogenous Poisson random measure, $Y$ can be expressed as

$$
Y(t)=\Sigma_{s \leq t} \Delta Y(s)=\int_{0}^{t} \int_{U} u \pi(d y, d s), \quad t \geq 0 .
$$

Hence,

$$
\begin{aligned}
& N_{1}(t)=\sum_{s \leq t} 1_{|\Delta Y(s)|<1} \Delta Y(s)=\int_{0}^{t} \int_{|u|<1} u \pi(d y, d s), \\
& N_{2}(t)=\Sigma_{s \leq t} 1_{|\Delta Y(s)| \geq 1} \Delta Y(s)=\int_{0}^{t} \int_{|u| \geq 1} u \pi(d y, d s) .
\end{aligned}
$$

Assume that the operator $\Psi(t), t \in[0, T]$, is a strongly measurable function taking values in the space of all bounded linear operator from $U$ to $E$. Let $0<\tau_{1}<\tau_{2}<\tau_{3}<\cdots \rightarrow \infty$ be the jump times for $N_{2}$ and $\Delta N_{2}\left(\tau_{k}\right)=\Delta Y\left(\tau_{k}\right)=Y\left(\tau_{k}\right)-$ $Y\left(\tau_{k}-\right), k=1,2, \ldots$. Then, the stochastic integral of $\Psi(t)$ with respect to jump process $N_{2}(t), t \geq 0$, can be defined as

$$
\int_{0}^{t} \Psi(s) d N_{2}(s)=\Sigma_{\tau_{k} \leq t} \Psi\left(\tau_{k}\right) \Delta N_{2}\left(\tau_{k}\right) .
$$

Since the operator $\Psi$ is taking values in $E$, it follows from the decomposition of $Y$ that the sum of sequences is finite. Hence the stochastic integral of the operator $\Psi$ with respect to $N_{2}$ is taking values in $E$. Moreover, the stochastic integral of the operator $\Psi(t), t \in[0, T]$, with respect to Lévy process $Y$ can be defined by

$$
\int_{0}^{t} \Psi(s) d Y(s)=\int_{0}^{t} \Psi(s) d N_{1}(s)+\int_{0}^{t} \Psi(s) d N_{2}(s)
$$

and takes values in $E$ as well (see [11] for more details).

Next, we recall some basic definitions and properties for general random dynamical systems, which are taken from [7]. Let $(H, d)$ be a complete separable metric space and $(\Omega, \mathscr{F}, \mathbb{P})$ be a probability space. 
Definition 4. $\left(\Omega, \mathscr{F}, \mathbb{P},(\theta)_{t \in \mathbb{R}}\right)$ is called a metric dynamical system if the mapping $\theta: \mathbb{R} \times \Omega \rightarrow \Omega$ is $(\mathscr{B}(\mathbb{R}) \times \mathscr{F}, \mathscr{F})$ measurable, $\theta_{0}=I, \theta_{s+t}=\theta_{s} \circ \theta_{t}$ for all $t, s \in R$, and $\theta_{t} \mathbb{P}=\mathbb{P}$ for all $t \in \mathbb{R}$.

Definition 5. A random dynamical system (RDS) with time $T$ on $(H, d)$ over $\left\{\theta_{t}\right\}$ on $\left(\Omega, \mathscr{F}, \mathbb{P},\left(\theta_{t}\right)_{t \in R}\right)$ is a $\left(\mathscr{B}\left(R^{+}\right) \times \mathfrak{F} \times\right.$ $\mathscr{B}(H), B(H))$-measurable map:

$$
\Pi: T \times H \times \Omega \longrightarrow H \times \Omega, \quad \Pi(t, s, \omega)=\left(S(t, \omega) x, \theta_{t} \omega\right)
$$

such that

(i) $S(0, \omega)=I d$ (identity on $H$ ) for any $\omega \in \Omega$,

(ii) (Cocycle property) $S(t+s, \omega)=S\left(t, \theta_{s} \omega\right) \circ S(s, \omega)$ for all $s, t \in T$ and $\omega \in \Omega$.

An RDS is said to be continuous or differentiable if for all $t \in T$, and an arbitrary outside outside $\mathbb{P}$-nullset $B \subset \Omega, w \in$ $B$ the map $S(t, \omega): H \rightarrow H$ is continuous or differentiable, respectively.

Assume that the bounded linear operator $A$ generates a $C_{0}$-semigroup $S=\left(e^{t A}\right)_{t \geq 0}$ on a Hilbert space $E$ and $Y$ defined on a filtered probability space $\left(\Omega, \mathscr{F},(\mathscr{F})_{t \geq 0}, \mathbb{P}\right)$ is a subordinator Lévy process taking values in a Hilbert space $U$.

Consider the following stochastic Langevin equation:

$$
\begin{gathered}
d X(t)=A X(t) d t+d Y(t), \quad t \geq t_{0}, \\
X\left(t_{0}\right)=x \in E .
\end{gathered}
$$

Definition 6. Let $x \in E$ be a square integrable $\mathscr{F}_{t_{0}}$-measurable random variable in $E$. A predicable process $X:\left[t_{0}, \infty\right) \times$ $\Omega \rightarrow E$ is called a mild solution of the Langevin equation (24) with initial data $\left(t_{0}, x\right)$ if it is an adapted $E$-valued stochastic process and satisfies

$$
X(t)=S\left(t-t_{0}\right) x+\int_{t_{0}}^{t} S(t-s) d Y(s), \quad t \geq t_{0} .
$$

It is well known that the Ornstein-Uhlenbeck process $X(t), t \geq 0$, has some important integrability. Here we need the Banach space to be of type $p$, for $p \in(1,2]$. First we recall the definition briefly (see [14] for more details).

Definition 7 (see [14]). For $p \in(1,2]$, the Banach space $E$ is called as type $p$, if and only if there exists a constant $K_{p}(E)>0$ such that for any finite sequence of symmetric independent identically distribution random variables $\varepsilon_{1}, \ldots, \varepsilon_{n}: \Omega \rightarrow$ $[-1,1], n \in \mathbb{N}$, and any finite sequence $x_{1}, \ldots, x_{n}$ from $E$, satisfying

$$
\mathbb{E}\left|\sum_{i=1}^{n} \varepsilon_{i} x_{i}\right|^{p} \leq K_{p}(E) \sum_{i=1}^{n}\left|x_{i}\right|^{p} .
$$

Moreover, if there exists a constant $L_{p}(E)>0$ such that for every $E$-valued martingale $\left\{M_{n}\right\}_{n=0}^{N}, N \in \mathbb{N}$, satisfying

$$
\sup _{n} \mathbb{E}\left|M_{n}\right|^{p} \leq L_{p}(E) \sum_{n=0}^{N} \mathbb{E}\left|M_{n}-M_{n-1}\right|^{p}, \quad M_{-1}=0,
$$

the separable Banach space $E$ is called a separable martingale type $p$-Banach space.

Lemma 8 (see [11, Corollary 8.1, Proposition 8.4]). Assume that $p \in(1,2], Z$ is a subordinator Lévy process from the class $\operatorname{Sub}(p), E$ is a separable type $p$-Banach, $U$ is a separable Hilbert space $U, E \subset U$, and $W=(W(t))_{t \geq 0}$ is a $U$-valued Wiener process.

Define the U-valued Lévy process as

$$
Y(t)=W(Z(t)), \quad t \geq 0,
$$

and define the process as

$$
X(t)=\int_{0}^{t} e^{(t-s) A} d Y(s)
$$

Then, with probability 1, for all $T>0$,

$$
\begin{aligned}
& \int_{0}^{T}|X(t)|_{E}^{p} d t<\infty, \\
& \int_{0}^{T}|X(t)|_{L^{4}}^{4} d t<\infty .
\end{aligned}
$$

We have the following existence and regularity results, which have been studied in $[2,11]$.

Theorem 9. Assume that $E=U, S=S(t), t \geq 0$ is the $C_{0}$ semigroup generated by the bounded linear operator $A$ in the space E. Then, if one of the following conditions is satisfied:

(i) $p \in(0,1]$ or

(ii) $p \in(1,2]$ and the Banach space $E$ is of separable martingale type p-Banach space,

the Langevin equation (24) admits one mild solution $X(t) \in E$, $t>0$. Moreover, if $p \in(1,2], S=S(t), t \geq 0$, is a $C_{0}$-group in the separable martingale type p-Banach space E, then the mild solution $X$ of the Langevin equation is a cádlág (rightcontinuous with left-hand side limits) process.

Proof. As $S=S(t), t \geq 0$, is a $C_{0}$-group in the separable martingale type $p$-Banach space $E$, the Hilbert space $H$ is the reproducing kernel Hilbert space of $W(1)$, and the embedding operator $i: H \hookrightarrow E$ satisfies the $\gamma$-radonifying property. The proof of Theorem 9 is just a simple application of Theorems 4.1 and 4.4 in [11].

\section{Cocycle Property of the Stochastic Boussinesq Equations}

In this section, we will show the existence, uniqueness, regularity, and the cocycle property of the stochastic Boussinesq equations (11).

It is well known that both $A_{1}$ and $A_{2}$ are positive definite, self-adjoint operators, and denote $D\left(A_{1}\right)$ and $D\left(A_{2}\right)$ to be the domains of $A_{1}$ and $A_{2}$, respectively. Hence, the domain of the operator $A$ can be represented as $D(A)=D\left(A_{1}\right) \times D\left(A_{2}\right)$. 
It follows from Lemma 2.2 in [7] that there exists positive numbers $\mu_{1}, \mu_{2}$, such that

$$
\begin{gathered}
\left(A_{1} u, u\right) \geq \mu_{1}\|u\|_{\left(L^{2}\right)^{2},}^{2} \\
\left(A_{2}(u, \theta),(u, \theta)\right) \geq \mu_{2}\|(u, \theta)\|^{2} .
\end{gathered}
$$

Let $\lambda=\min \left(\mu_{1}, \mu_{2}\right)$. Then

$$
(A U, U) \geq \lambda\|U\|^{2} .
$$

For any arbitrary $U, V, W \in \mathbb{V}$, we can define the following trilinear form $b: U \times V \times W \rightarrow \mathbb{R}$ by

$$
\begin{gathered}
b(u, v, w)=\langle B(u, v), w\rangle, \\
b(U, V, W)=b_{1}(u, v, w)+b_{2}(u, \widetilde{v}, \widetilde{w}), \\
b_{1}(u, v, w)=\int_{D} \sigma_{i, j}^{2} u_{i} \frac{\partial v_{j}}{\partial x_{i}} w_{j} d x, \\
b_{2}(u, \widetilde{v}, \widetilde{w})=\int_{D} \sigma_{i}^{2} u_{i} \frac{\partial \widetilde{v}_{j}}{\partial x_{i}} \widetilde{w}_{j} d x .
\end{gathered}
$$

We have the following results.

Lemma 10 (see [7, Lemma 2.3]). If $U, V, W \in \mathbb{V}$, then

$$
\begin{gathered}
b(U, V, W)=-b(U, W, V), \\
(B(V, U), U)=b(V, U, U)=0 .
\end{gathered}
$$

Lemma 11 (see [7, Lemma 2.4]). There exists a constant $c_{B}>$ 0 such that if $u \in V_{1}, \theta, \eta \in V_{2}, \phi=(u, \theta)$, then

(1) $\left|b_{1}(u, v, w)\right| \leq c_{B}\|u\|_{H^{1}}\|v\|_{H^{2}}\|w\|, u \in V, v \in D(A)$, $w \in H$,

(2) $\left|b_{1}(u, v, w)\right| \leq c_{B}\|u\|_{L^{2}}^{1 / 2}\|u\|_{H^{1}}^{1 / 2}\|v\|_{H^{2}}\|w\|^{1 / 2}\|w\|_{H^{1}}^{1 / 2}, u \in$ $V, v \in D(A), w \in V$,

(3) $\left|b_{1}(u, v, u)\right| \leq c_{B}\|u\|_{H^{1}}\|v\|_{H^{1}}\|v\|, u \in V, v \in V$,

(4) $\left|b_{2}(u, \theta, w)\right| \leq c_{B}\|u\|^{1 / 2}\|u\|_{H^{1}}^{1 / 2}\|\theta\|_{H^{1}}\|w\|^{1 / 2}\|w\|_{H^{1}}^{1 / 2}, u \in$ $V, \theta \in V, w \in V$,

(5) $\left|b_{2}(u, \theta, w)\right| \leq c_{B}\|u\|\|\theta\|_{H^{2}}\|w\|_{H^{1}}, u \in H, \theta \in D(A)$, $w \in V$.

Definition 12. An $H$-valued $\left(\mathscr{F}_{t}\right)_{t \geq 0}$ adapted and $\mathbf{H}^{4,2}(D)$ valued cádlág process $u(t)(t \geq 0)$ is considered as a solution to (11), if for each $T>0$,

$$
\sup _{0 \leq t \leq T}|U(t)|_{H}^{2}+\int_{0}^{T}|U(t)|_{\mathbb{L}^{4}(D)}^{4} d t<\infty, \quad \text { a.s. }
$$

and for any $\psi \in V \cap \mathbf{H}^{2,2}(D)$, and for any $t>0, \mathbb{P}$-a.s.,

$$
\begin{aligned}
& (U(t), \psi)-\left(U_{0}, \psi\right)-\int_{0}^{t}(U(s), \Delta \psi) d s \\
& \quad+\int_{0}^{t}(B(U, U), \psi(s)) d s+\int_{0}^{t}(R(U), \psi) d s=(\psi, Y(t)) .
\end{aligned}
$$

Denote

$\mathscr{H}^{1,2}(0, T)=\left\{\right.$ the space of all functions $v \in L^{2}(0, T ; V)$

$$
\left.\cap \mathbb{M}^{2,2}(D) \text { satisfying } v^{\prime} \in L^{2}\left(0, T ; V^{\prime}\right)\right\} \text {. }
$$

Lemma 13. Assume that $z \in L^{4}\left(0, T ; \mathbb{L}^{4}(D)\right), g \in L^{2}\left(0, T, V^{\prime}\right)$, and $v_{0} \in H$. Then there exists a unique $v \in \mathscr{H}^{1,2}(0, T)$ such that

$$
\begin{gathered}
\frac{d v}{d t}+A v+B(v, z)+B(z, v)+B(v, v)=g, \quad t \geq 0, \\
v(0)=v_{0} .
\end{gathered}
$$

Moreover,

$$
\begin{gathered}
\sup _{t \in[0, T]}|v(t)|^{2} \leq K^{2} L^{2}, \quad \int_{0}^{T}|\nabla v(t)|^{2} d t \leq M^{2}, \\
\int_{0}^{T}\left|v^{\prime}(t)\right|_{V^{\prime}}^{2} d t \leq N^{2}, \quad \int_{0}^{T}|v(t)|_{\mathbb{L}^{4}(D)}^{4} d t \leq 2 T^{1 / 2} K^{3} L^{3} M,
\end{gathered}
$$

where

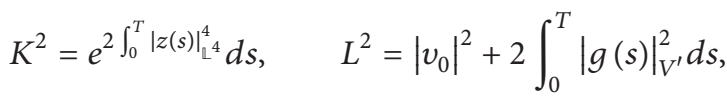

$$
\begin{aligned}
& M^{2}=\left|v_{0}\right|^{2}+9 K L \int_{0}^{T}|z(t)|_{L^{4}\left(0, T, \mathbb{L}^{4}(D)\right)}^{2}+\frac{T^{1 / 4}}{\sqrt{2}} K^{3 / 2} L^{1 / 2},
\end{aligned}
$$

and the mapping $L^{2}\left(0, T, V^{\prime}\right) \times H \ni\left(g_{0}, v_{0}\right) \mapsto v \in \mathscr{H}^{1,2}(0, T)$ is analytic.

Proof. It can be shown by the same approach as the one in Proposition 8.7 in [11].

Lemma 14 (see [2, Proposition 10.1]). Let $u:[0, T] \rightarrow B$ be a continuous function whose left derivative

$$
\frac{d^{-} u}{d t}\left(t_{0}\right)=\lim _{\epsilon \rightarrow 0, \epsilon<0} \frac{u\left(t_{0}+\epsilon\right)-u\left(t_{0}\right)}{\epsilon}
$$

exists at $t_{0} \in[0, T]$. Then the function $\gamma(t)=|u(t)|_{B}, t \in[0, T]$, is left differentiable at $t_{0}$ and

$$
\frac{d^{-} \gamma}{d t}\left(t_{0}\right)=\min \left\{\left\langle x^{*}, \frac{d^{-} u}{d t}\left(t_{0}\right)\right\rangle: x^{*} \in \partial\left|u\left(t_{0}\right)\right|_{B}\right\} .
$$

In order to apply the Yosida approximation for the solution of (11), we need to introduce some definitions of dissipative mapping (operator) (see [17] for details).

Definition 15. Let $\left(B,|\cdot|_{B}\right)$ be a separable Banach space, $B^{*}$ be the dual space of $B$. The subdifferential $\partial|x|_{B}$ of norm $|\cdot|_{B}$ at $x \in B$ is defined by the formula

$$
\partial|x|_{B}:=\left\{x^{*} \in B^{*}:|x+y|_{B}-|x|_{B} \geq\left(x^{*}, y\right), \forall y \in B\right\} .
$$


A mapping $F: D(F) \subset B \rightarrow B$ is said to be dissipative, if for any $x, y \in D(F)$, there exists $z^{*} \in \partial|x-y|_{B}$ such that

$$
\left\langle z^{*}, F(x)-F(y)\right\rangle \leq 0 .
$$

A dissipative mapping $F: D(F) \subset B \rightarrow B$ is called an $m$-dissipative mapping or maximal dissipative if the image of $I-\lambda F$ is equal to the whole space $B$ for some $\lambda>0$ (and then for any $\lambda>0$ ), that is,

$$
\text { range }(I-\lambda A)=B, \quad \text { for some } \lambda>0 \text {. }
$$

Assume that $F$ is an $m$-dissipative mapping. Then its resolvent $J_{\alpha}$ and respectively the Yosida approximations $F_{\alpha}$, $\alpha>0$, are defined by

$$
\begin{gathered}
J_{\alpha} x=(I-\alpha F)^{-1} x \in \operatorname{dom} F \\
F_{\alpha} x=\frac{1}{\alpha}\left(J_{\alpha} x-x\right), \quad \forall x \in \operatorname{dom} J_{\alpha}=\operatorname{range}(I-\alpha F) .
\end{gathered}
$$

Lemma 16 (see [2, Proposition 10.2]). Let $F: D(F) \rightarrow B$ be an $m$-dissipative mapping on $B$. Then

(1) for all $\alpha>0$ and $x, y \in B,\left|J_{\alpha}(x)-J_{\alpha}(y)\right|_{B} \leq|x-y|_{B}$;

(2) the mapping $F_{\alpha}, \alpha>0$, are dissipative and Lipschitz continuous:

$$
\left|F_{\alpha}(x)-F_{\alpha}(y)\right|_{B} \leq \frac{2}{\alpha}|x-y|_{B}, \quad \forall x, y \in B .
$$

Moreover, $\left|F_{\alpha}(x)\right|_{B} \leq|F(x)|_{B}$, for all $x \in D(F)$; and

(3) $\lim _{\alpha \rightarrow 0} F_{\alpha}(x)=x$, for all $x \in \overline{D(F)}$.

The following theorem is one of the main results of this paper, which will be proved by applying the well-known Yosida approach.

Theorem 17. For every $u_{0} \in H$, under Assumption 1, the stochastic Boussinesq system (11) admits a unique cádlág mild solution $u(t)$.

Proof. Denote $Z_{A}(\omega)$ to be the stationary solution of Langevin equation (24). Let $V=U-Z_{A}$. Then (11) is converted into the following evolution equation with random coefficients:

$$
\begin{gathered}
d V=\left[A V+B\left(V+Z_{A}, V+Z_{A}\right)+R\left(V+Z_{A}\right)\right] d t, \quad t \geq 0, \\
V(0)=U_{0},
\end{gathered}
$$

where $(A, D(A))$ generates an analytic $C_{0}$-semigroup $S$ (see Section 2.2 in [2]). It follows from the proof of Theorem 10.1 in [2] that, for $\alpha>0, \beta>0$, and sufficiently small $\eta$, the mappings $A+\eta$ and $B(\cdot, \cdot)+R(\cdot)+\eta$ are $m$-dissipative. Hence, the Yosida approximations of the $m$-dissipative mappings $A+$ $\eta$ and $B(\cdot, \cdot)+R(\cdot)+\eta$ can be respectively denoted by

$$
\begin{gathered}
(A+\eta)_{\beta}=\frac{1}{\beta}\left((I-\beta(A+\eta))^{-1}-I\right), \\
((B+R)+\eta)_{\alpha}=\frac{1}{\alpha}\left((I-\alpha((B+R)+\eta))^{-1}-I\right) .
\end{gathered}
$$

Now consider the following random approximate equation:

$$
\begin{gathered}
\frac{d^{-}}{d t} Y_{\alpha, \beta}(t)=(A+\eta)_{\beta} Y_{\alpha, \beta}+(B+R+\eta)_{\alpha}\left(Y_{\alpha, \beta}+Z_{A}(t-)\right) \\
-2 \eta Y_{\alpha, \beta}-\eta Z_{A}(t-), \\
Y_{\alpha, \beta}(0)=U_{0} .
\end{gathered}
$$

It is easy to verify that $\left((A+\eta)_{\beta}, D\left((A+\eta)_{\beta}\right)\right)$ generates an analytic $C_{0}$-semigroup $S_{\beta}$. Notice that the Yosida approximate operators are Lipschitz. Therefore the random approximation equation (50) has a unique continuous solution $Y_{\alpha, \beta}$.

Next we will show that

$$
\lim _{\alpha \rightarrow 0}\left[\lim _{\beta \rightarrow 0} Y_{\alpha, \beta}(t)\right]=Y(t), \quad t \geq 0,
$$

in $H$, and this limit is actually the mild solution of stochastic Boussinesq equation (48).

For the sake of simplification, we just present the estimations when $\eta=0$, and the remaining estimates can be obtained by the similar arguments for $\eta \neq 0$.

Let $Y_{\alpha}$ be the solution of the integral equation:

$$
\begin{array}{rl}
Y_{\alpha}(t)=S(t) U_{0}+\int_{0}^{t} & S(t-s) \\
& \times\left(B\left(Y_{\alpha}(s)+Z_{A}(s-), Y_{\alpha}(s)+Z_{A}(s-)\right)\right. \\
& \left.+R\left(Y_{\alpha}(s)+Z_{A}(s-)\right)\right)_{\alpha} d s .
\end{array}
$$

Notice that the operator $(B(\cdot, \cdot)+R(\cdot))_{\alpha}$ is Lipschitz continuous and $Z_{A}$ is cádlág. Hence, there exists a solution of random approximate equation (50), which is continuous in $H$.

For $\alpha>0$ and $\beta>0$, direct computation implies

$$
\begin{gathered}
Y_{\alpha}-Y_{\alpha, \beta}=S(t) U_{0}-S_{\beta}(t) \\
+\int_{0}^{t}\left[S(t-s)-S_{\beta}(t-s)\right] \\
\times\left[B\left(Y_{\alpha}(s)+Z_{A}(s-), Y_{\alpha}(s)+Z_{A}(s-)\right)\right. \\
\left.+R\left(Y_{\alpha}(s)+Z_{A}(s-)\right)\right]_{\alpha} d s \\
+\int_{0}^{t}\left[S_{\beta}(t-s)\right] \\
\times\left[B\left(Y_{\alpha}(s)+Z_{A}(s-), Y_{\alpha}(s)+Z_{A}(s-)\right)\right. \\
\left.+R\left(Y_{\alpha}(s)+Z_{A}(s-)\right)\right]_{\alpha} \\
-\left[B \left(Y_{\alpha, \beta}(s)+Z_{A}(s-),\right.\right. \\
\left.Y_{\alpha, \beta}(s)+Z_{A}(s-)\right) \\
\left.\left.+R\left(Y_{\alpha, \beta}(s)+Z_{A}(s-)\right)\right]_{\alpha}\right] d s .
\end{gathered}
$$


Since both $A$ and $B+R$ are $m$-dissipative. Therefore, there exists constant $M, \omega$, and $C_{\alpha}$ such that for all $t \geq 0, V, W \in H$,

$$
\begin{gathered}
\left\|S_{\beta}(t)\right\|_{L(H, H)} \leq M e^{\omega t}, \\
\left|[B(V)+R(V)]_{\alpha}-[B(U)+R(U)]_{\alpha}\right| \leq C_{\alpha}|V-U|_{H} .
\end{gathered}
$$

Then

$$
\begin{gathered}
\left|Y_{\alpha}(t)-Y_{\alpha, \beta}(t)\right| \\
\leq\left|S(t) U_{0}-S_{\beta}(t) U_{0}\right|+M C_{\alpha} \int_{0}^{t} e^{\omega(t-s)}\left|Y_{\alpha}(s)-Y_{\alpha, \beta}(s)\right| d s \\
+\int_{0}^{t} \mid\left[S_{\beta}(t-s)-S(t-s)\right] \\
\times\left[B\left(Y_{\alpha, \beta}(s)+Z_{A}(s-), Y_{\alpha, \beta}(s)+Z_{A}(s-)\right)\right. \\
\left.+R\left(Y_{\alpha, \beta}(s)+Z_{A}(s-)\right)\right]_{\alpha} \mid d s .
\end{gathered}
$$

By the Hille-Yosida theorem, it follows that

$$
S_{\beta}(t) U_{0} \longrightarrow S(t) U_{0}, \quad \text { as } \beta \longrightarrow 0
$$

uniformly in $t$ on compact subsets $U_{0}$ of $H$.

Hence, it follows that

$$
\left|Y_{\alpha}(t)-Y_{\alpha, \beta}(t)\right| \leq M C_{\alpha} \int_{0}^{t}\left|Y_{\alpha}(s)-Y_{\alpha, \beta}(s)\right| d s
$$

uniformly on bounded intervals as $\beta \rightarrow 0$.

By Gronwall inequality, we have

$$
\limsup _{\beta \rightarrow 0}\left|Y_{\alpha \leq T}(t)-Y_{\alpha, \beta}(t)\right|=0, \quad \forall T<\infty
$$

\section{By Lemma 14,}

$$
\begin{gathered}
\frac{d^{-}}{d t}\left|Y_{\alpha, \beta}(t)\right| \\
=\min \left\{\left\langle x^{*}, \frac{d^{-}}{d t} Y_{\alpha, \beta}(t)\right\rangle: x^{*} \in \partial\left|Y_{\alpha, \beta}(t)\right|\right\} \\
=\min \left\{\left\langlex^{*}, A_{\beta} Y_{\alpha, \beta}(t)\right.\right. \\
+\left[B\left(Y_{\alpha, \beta}(s)+Z_{A}(s-), Y_{\alpha, \beta}(s)+Z_{A}(s-)\right)\right. \\
\left.\left.+R\left(Y_{\alpha, \beta}(s)+Z_{A}(s-)\right)\right]_{\alpha}\right\rangle: \\
\left.x^{*} \in \partial\left|Y_{\alpha, \beta}(t)\right|\right\} .
\end{gathered}
$$

Recalling that both $A_{\beta}$ and $[B(\cdot, \cdot)+R(\cdot)]$ are $m$-dissipative and $A_{\beta}$ is linear, we obtain

$$
\begin{aligned}
\frac{d^{-}}{d t}\left|Y_{\alpha, \beta}(t)\right| \leq \mid & B\left(Y_{\alpha, \beta}(t)+Z_{A}(t-), Y_{\alpha, \beta}(s)+Z_{A}(t-)\right) \\
+ & \left.R\left(Y_{\alpha, \beta}(t)+Z_{A}(t-)\right)\right]_{\alpha} \mid \\
\leq \mid B( & \left.Y_{\alpha, \beta}(t)+Z_{A}(t-), Y_{\alpha, \beta}(s)+Z_{A}(t-)\right) \\
+ & R\left(Y_{\alpha, \beta}(t)+Z_{A}(t-)\right) \mid,
\end{aligned}
$$

that is,

$$
\begin{array}{r}
\left|Y_{\alpha, \beta}(t)\right| \leq\left|U_{0}\right|+\int_{0}^{t} \mid B\left(Y_{\alpha, \beta}(s)+Z_{A}\left(s^{-}\right), Y_{\alpha, \beta}(s)+Z_{A}\left(s^{-}\right)\right) \\
+R\left(Y_{\alpha, \beta}(s)+Z_{A}\left(s^{-}\right)\right) \mid d s, \quad t \geq 0 .
\end{array}
$$

It follows from the estimate (58) that, for any $\alpha>0$, and $t \epsilon$ $[0, T]$,

$$
\begin{aligned}
&\left|Y_{\alpha}(t)\right| \leq\left|U_{0}\right|+\int_{0}^{t} \mid {\left[B\left(Y_{\alpha, \beta}(s)+Z_{A}(s-), Y_{\alpha, \beta}(s)+Z_{A}(s-)\right)\right.} \\
&+R\left(Y_{\alpha, \beta}(s)+Z_{A}(s-)\right) \mid d s .
\end{aligned}
$$

Similarly, by Lemma 16 , for $t \in[0, T]$,

$$
\begin{aligned}
\frac{1}{2} \frac{d^{-}}{d t}\left|Y_{\alpha, \beta}(t)-Y_{\gamma, \beta}(t)\right|^{2} \\
=\left\langle\frac{d^{-}}{d t}\left(Y_{\alpha, \beta}(t)-Y_{\gamma, \beta}(t)\right), Y_{\alpha, \beta}(t)-Y_{\gamma, \beta}(t)\right\rangle \\
=\left\langle\left(A_{\beta} Y_{\alpha, \beta}(t)-A_{\beta} Y_{\gamma, \beta}(t)\right)+\left[(B+R)_{\alpha}\right]\right. \\
\times\left(Y_{\alpha, \beta}(t)+Z_{A}(\omega)(t-)\right)-\left[(B+R)_{\gamma}\right] \\
\left.\quad \times\left(Y_{\gamma, \beta}(t)+Z_{A}(\omega)(t-)\right), Y_{\alpha, \beta}(t)-Y_{\gamma, \beta}(t)\right\rangle \\
\leq\left\langle\left[(B+R)_{\alpha}\right]\left(Y_{\alpha, \beta}(t)+Z_{A}(\omega)(t-)\right)-\left[(B+R)_{\gamma}\right]\right. \\
\left.\quad \times\left(Y_{\gamma, \beta}(t)+Z_{A}(\omega)(t-)\right), Y_{\alpha, \beta}(t)-Y_{\gamma, \beta}(t)\right\rangle \\
\leq(\gamma+\alpha)\left[\left|(B+R)_{\alpha}\left(Y_{\alpha, \beta}(t)+Z_{A}(\omega)(t-)\right)\right|\right. \\
\left.\quad+\left|(B+R)_{\gamma}\left(Y_{\gamma, \beta}(t)+Z_{A}(\omega)(t-)\right)\right|\right]^{2} \\
\leq(\gamma+\alpha)\left[\left|(B+R)\left(Y_{\alpha, \beta}(t)+Z_{A}(\omega)(t-)\right)\right|\right. \\
\left.\quad+\left|(B+R)\left(Y_{\gamma, \beta}(t)+Z_{A}(\omega)(t-)\right)\right|\right]^{2} .
\end{aligned}
$$

By the dissipation of the operators $A, B$, and $R$ and estimates (63), there exists a constant $C>0$ such that

$$
\frac{1}{2} \frac{d^{-}}{d t}\left|Y_{\alpha, \beta}(t)-Y_{\gamma, \beta}(t)\right|^{2} \leq C(\alpha+\gamma), \quad t \in[0, T] .
$$


Therefore

$$
\left|Y_{\alpha, \beta}(t)-Y_{\gamma, \beta}(t)\right|^{2} \leq 2 C(\alpha+\gamma) T, \quad t \in[0, T] .
$$

By the estimate (58),

$$
\left|Y_{\alpha}(t)-Y_{\gamma}(t)\right|^{2} \leq 2 C(\alpha+\gamma) T, \quad t \in[0, T] .
$$

Thus, $Y_{\alpha}(t) \rightarrow Y(t)$ in $H$ uniformly on $[0, T]$ as $\alpha \rightarrow 0$.

Next, we are going to show that the solution $Y_{\alpha}$ of the Yosida approximations equation is a mild solution:

$$
\begin{array}{r}
Y_{\alpha}(t)=S(t) U_{0}+\int_{0}^{t} S(t-s)(B+R)_{\alpha}\left(Y_{\alpha}(s)+Z_{A}(s)\right) d s, \\
t \in[0, T] .
\end{array}
$$

By the reflexivity of $H^{1}$ and the estimate $\left\|Y_{\alpha}(t)\right\|_{H^{1}} \leq$ $C_{2}\left\|U_{0}\right\|_{H^{1}}, t \in[0, T], \alpha>0$, there exists a subsequence $\left\{Y_{\alpha, n}\right\}$, which converges weakly in $H^{1}$ and weakly converges to the function $Y(t)$ in $H^{1}$. Since $\left\{Y_{\alpha, n}(t)\right\}$ is strong convergent in $L^{2}$, and

$$
\|Y(t)\|_{H^{1}} \leq C_{2}\left\|U_{0}\right\|_{H^{1}}, \quad t \in[0, T]
$$

Let $h \in L^{2}$, then

$$
\begin{aligned}
& \left\langle Y_{\alpha}(t), h\right\rangle_{L^{2}} \\
& =\left\langle S(t) U_{0}, h\right\rangle_{L^{2}} \\
& \quad+\int_{0}^{t}\left\langle(B+R) J_{\alpha}\left(Y_{\alpha}(s)+Z_{A}(s)\right), S^{*}(t-s) h\right\rangle_{L^{2}} d s .
\end{aligned}
$$

Moreover

$$
J_{\alpha}\left(Y_{\alpha}(s)+Z_{A}(s)\right) \longrightarrow Y(s)+Z_{A}(s), \quad \text { as } \alpha \longrightarrow 0 \text {. }
$$

Notice that $(B+R)\left(J_{\alpha}\left(Y_{\alpha}(s)+Z_{A}(s)\right) \rightarrow(B+R)\left(Y_{\alpha}(s)+Z_{A}(s)\right)\right.$ weakly converges in $L^{2}$. So, letting $\alpha \rightarrow 0$, we obtain

$$
\begin{aligned}
\langle Y(t), h\rangle_{L^{2}}= & \left\langle S(t) U_{0}, h\right\rangle_{L^{2}} \\
& +\int_{0}^{t}\left\langle\left(S(t-s)(B+R)\left(Y(s)+Z_{A}(s)\right), h\right\rangle_{L^{2}} d s .\right.
\end{aligned}
$$

It follows from the arbitrariness of $h$ that

$$
Y(t)=S(t) U_{0}+\int_{0}^{t} S(t-s)(B+R)\left(Y_{\alpha}(s)+Z_{A}(s)\right) d s,
$$$$
t \in[0, T]
$$

Thus, $Y(t)$ is a mild solution of random Boussinesq equation (50).
Theorem 18. For any $U_{0} \in H$, the $\operatorname{map} \varphi: \mathbb{T} \times \Omega \times H \rightarrow H$ defined by the solution of stochastic Boussinesq equation (11) as $U(t)=\Phi\left(t, \vartheta_{t}(\omega)\right) U_{0}$ has the cocycle property; that is, the solution of stochastic Boussinesq equation (11) generates a random dynamical system $\left(\Omega, \mathscr{F}, \mathbb{P},\left(\vartheta_{t}\right)_{t \geq 0}, \Phi\right)$.

Proof. From Theorem 17, stochastic Boussinesq equation (11) admits a unique solution $V(t, Z(\omega)(t), x)$. Define the map

$$
\begin{gathered}
\Phi: \mathbb{R}^{+} \times \Omega \times H \longrightarrow H \\
\Phi(t, \omega) x=V(t \cdot Z(\omega)(t))(x-Z(\omega)(0))+Z(\omega)(t+s) .
\end{gathered}
$$

(i) By the similar argument of Theorem 17, every solution $Y_{\alpha}(t)$ of the Yosida approximation equation (50) is measurable. Notice that $Y_{\alpha}(t) \rightarrow Y(t)$ uniformly as $\alpha \rightarrow 0$. Hence, the limit function $Y(t)$ is also measurable. Thus, the mapping $\Phi$ is measurable.

(ii) Obviously, $\Phi(0, \omega)=I$.

(iii) It suffices to verify that the cocycle property holds for the mapping $\Phi$, that is,

$$
\begin{aligned}
\Phi(t+s, \omega) x= & V\left(t+s, Z_{A}(\omega)(t+s)\right)\left(x-Z_{A}(\omega)(0)\right) \\
& +Z_{A}(\omega)(t+s) .
\end{aligned}
$$

In fact, recalling that $Z_{A}(\omega)(s)=Z_{A}\left(\theta_{s} \omega\right)(0)$, it follows that

$$
\begin{aligned}
\Phi\left(t, \theta_{s} \omega\right)[\Phi(s, \omega) x] \\
=V\left(t, Z_{A}\left(\theta_{s} \omega\right)(t)\right)\left(\Phi(s, \omega) x-Z_{A}\left(\theta_{s} \omega\right)(0)\right) \\
\quad+Z_{A}\left(\theta_{s} \omega\right)(t) \\
=V\left(t, Z_{A}\left(\theta_{s} \omega\right)(t)\right) \\
\quad \times\left[V\left(s, Z_{A}(\omega)(s)\right)\left(x-Z_{A}(\omega)(0)\right)+Z(\omega)(s)\right. \\
\left.\quad-Z\left(\theta_{s} \omega\right)(0)\right]+Z\left(\theta_{s} \omega\right)(t) \\
=V\left(t, Z_{A}\left(\theta_{s} \omega\right)(t)\right) V\left(s, Z_{A}(\omega)(s)\right) \\
\quad \times\left(x-Z_{A}(\omega)(0)\right)+Z_{A}\left(\theta_{s} \omega\right)(t) \\
=\quad V_{1}(t) .
\end{aligned}
$$

Moreover,

$$
\begin{aligned}
& V\left(t+s, Z_{A}(\omega)(t+s)\right)\left(x-Z_{A}(\omega)(0)\right) \\
& \quad=V\left(t, Z_{A}\left(\theta_{s} \omega\right)(t)\right) V\left(s, Z_{A}(\omega)(s)\right)\left(x-Z_{A}(\omega)(0)\right) \\
& \quad=V_{2}(t)
\end{aligned}
$$

Since

$V\left(0, Z_{A}\left(\theta_{s} \omega\right)(0)\right)\left(x-Z_{A}\left(\theta_{s} \omega\right)(0)\right)=x-Z_{A}\left(\theta_{s} \omega\right)(0)$. 
Thus,

$$
\begin{aligned}
V_{1}(0)= & V\left(s, Z_{A}(\omega)(s)\right)\left(x-Z_{A}(\omega)(0)\right) \\
= & V\left(0, Z_{A}\left(\theta_{s} \omega\right)(0)\right) V\left(s, Z_{A}(\omega)(s)\right) \\
& \times\left(x-Z_{A}(\omega)(0)\right)=V_{2}(0), \\
\frac{d V_{1}(t)}{d t} & =\frac{d V\left((t+s), Z_{A}(\omega)\right)}{d t}(t+s) .
\end{aligned}
$$

Therefore, we obtain

$$
\begin{aligned}
\frac{d V_{1}(t)}{d t}+ & A V_{1}(t) \\
& +B\left(V_{1}(t)+Z_{A}(\omega)(t+s), V_{1}(t)+Z_{A}(\omega)(t+s)\right) \\
= & -R\left(V_{1}(t)+Z_{A}\left(\theta_{t+s} \omega\right)\right), \\
\frac{d V_{2}(t)}{d t}+ & A V_{2}(t) \\
& +B\left(V_{2}(t)+Z_{A}\left(\theta_{s} \omega\right)(t), V_{2}(t)+Z_{A}\left(\theta_{s} \omega\right)(t)\right) \\
= & -R\left(V_{2}(t)+Z_{A}\left(\theta_{s} \omega\right)(t)\right) .
\end{aligned}
$$

The uniqueness of the solution implies that almost surely $V_{1}(t)=V_{2}(t)$ holds, that is,

$$
\Phi\left(t, \theta_{s} \omega\right)[\Phi(s, \omega) x]=\Phi\left(t+s, \theta_{t+s}(\omega)\right) x
$$

Thus, the cocycle property for the mapping $\Phi$ holds.

By the definition of random dynamical systems [18], the solution mapping of the stochastic Boussinesq equation (11) generates a random dynamical system $\Phi$. Thus, the proof of Theorem 18 is complete.

\section{Discussion}

In Section 3, we have studied the long-time behavior of stochastic Boussinesq equations (1) driven by subordinator Lévy noise and have shown the cocycle property of random dynamical systems generated by the mild solution of stochastic Boussinesq equation (1). To prove the existence of random attractor, it suffices to show the existence of random absorbing set and the compactness of random dynamical system $\Phi$, we refer the similar argument to [13].

Here, we are also interested in the stochastic Boussinesq equations driven by Poisson noise and Wiener noise, and we are trying to show the existence of random dynamical systems. To the end, we consider the following stochastic Boussinesq equations driven by Lévy noises followed as

$$
\begin{aligned}
& \frac{\partial u}{\partial t}+(u \cdot \nabla) u-v \Delta u+\nabla p \\
& =\theta e_{2}+b_{1} d t+d W^{1}(t)+\int_{X} f(x) \widetilde{N}^{1}(d t, d x), \\
& \frac{\partial \theta}{\partial t}+(u \cdot \nabla) \theta-k \Delta \theta \\
& =u_{2}+b_{2} d t+d W^{2}(t)+\int_{X} g(x) \widetilde{N}_{2}(d t, d x), \\
& \quad \nabla \cdot u=0, \\
& \left.u\right|_{\partial D}=0, \quad u(0)=u_{0}, \quad \theta(0)=\theta_{0},
\end{aligned}
$$

where $W^{1}(\cdot)$ and $W^{2}(\cdot)$ are $H$-valued Brownian motion, $b_{1}$ and $b_{2}$ are constants vector in $H, f$ and $g$ are measurable mappings from some measurable space $X$ to $H$, and $\widetilde{N}_{1}$ and $\widetilde{N}_{2}$ are compensated Poisson measure on $[0, \infty) \times X$ with intensity measure $n v_{1}$ and $n v_{2}$, respectively, where $v_{1}$ and $v_{2}$ are $\sigma$-finite measure on $\mathscr{B}(X), f(x)$, and $g(x)$ satisfying

$$
\begin{gathered}
\int_{U}|f(x)|^{2} e^{\alpha|f(x)|} v(d x)<\infty, \\
\int_{U}|g(x)|^{2} e^{\beta|g(x)|} v(d x)<\infty, \quad \forall \alpha>0, \quad \forall \beta>0 .
\end{gathered}
$$

Let $D([0, T], H)$ be the space of all cádlág paths from $[0, T]$ to $H$ endowed with the uniform convergence topology. Since there are finite jumps when the character measure $\lambda(Z)<\infty$, we can rearrange the jump time of $N(d t, d x)$ as $\sigma_{1}(\omega)<\sigma_{2}(\omega)<\cdots$. Since there is no jump on the interval $\left[0, \sigma_{1}(\omega)\right)$, just as the approach in [19], we can apply Banach fixed point theorem to prove that there exists a unique solution $\phi(t)$ in $L^{2}\left(\left[0, \sigma_{1}(\omega)\right) ; V\right) \cap D\left(\left[0, \sigma_{1}(\omega)\right) ; H\right)$. Define

$$
\phi^{(1)}(t)= \begin{cases}\phi(t), & 0 \leq t<\sigma_{1}(\omega), \\ \phi\left(\sigma_{1}-\right)+f\left(\phi\left(\sigma_{1}-\right), P_{\sigma_{1}}\right), & t=\sigma_{1}(\omega) .\end{cases}
$$

On $\left[\sigma_{1}(\omega), \sigma_{2}(\omega)\right)$, define

$$
\begin{aligned}
\widetilde{\phi}_{0} & =\phi^{(1)}\left(\sigma_{1}\right) 1_{\left(\sigma_{1}<\infty\right)}, \\
\widetilde{\sigma}_{2} & =\left(\sigma_{2}-\sigma_{1}\right) 1_{\left(\sigma_{1}<\infty\right)}+\infty 1_{\left(\sigma_{1}=\infty\right)}, \\
\widetilde{F}_{t} & =\mathscr{F}_{\sigma_{1}+t}, \quad \widetilde{P}_{t}=\left(\theta_{\sigma_{1}} P\right)_{t} 1_{\left(\sigma_{1}<\infty\right)} .
\end{aligned}
$$

Similar to the argument in [11], since $P_{t}$ is stationary Poisson point process on $R^{+} \times Z$ with intensity measure $\lambda(d x) d t$, 
then $\widetilde{P}_{t}$ is also a stable Poisson point process on $R^{+} \times Z$ with intensity measure $\lambda(d x) d t$. Define

$$
\begin{aligned}
& \phi^{(2)}(t) \\
& = \begin{cases}\phi^{(1)}(t), & 0 \leq t<\sigma_{1}(\omega), \\
\widetilde{\phi}^{(2)}\left(t-\sigma_{1}\right), & \sigma_{1}(\omega)<t<\sigma_{2}(\omega), \\
\widetilde{\phi}^{(2)}\left(\left(\sigma_{2}-\sigma_{1}\right)-\right) & \\
+f\left(\widetilde{\phi}^{(2)}\left(\left(\sigma_{2}-\sigma_{1}\right)-\right), P_{\sigma_{2}}\right), & t=\sigma_{2}(\omega),\end{cases} \\
& \phi^{(n)}(t) \begin{array}{ll}
\phi^{(n-1)}(t), \\
\widetilde{\phi}^{(n)}\left(t-\sigma_{n-1}\right), & t<\sigma_{n-1}(\omega), \\
\widetilde{\phi}^{(n)}\left(\left(\sigma_{n}-\sigma_{n-1}\right)-\right) & \sigma_{n-1}(\omega)<t<\sigma_{n}(\omega), \\
+f\left(\widetilde{\phi}^{(n)}\left(\left(\sigma_{n}-\sigma_{n-1}\right)-\right), P_{\sigma_{n}}\right), & t=\sigma_{n} .
\end{array}
\end{aligned}
$$

Hence, $\phi^{(n)}(t)$ is cádlág on $[0, T]$ such that $B\left(\phi^{(n)}, \phi^{(n)}\right) \in H$ and $A_{p}\left(\phi^{(n)} \in H, P\right.$ a.s. for all $t \geq 0$, and

$$
\begin{aligned}
& P\left(\int _ { 0 } ^ { t } \left[|\phi(s)|+\left|B\left(\phi(s)+z_{A}(s), \phi(s)+z_{A}(s)\right)\right|\right.\right. \\
& \left.\left.\quad+2 \mu_{0}\left|R\left(\phi(s)+z_{A}(s)\right)\right|\right] d s<\infty\right)=1, \quad \forall t>0 .
\end{aligned}
$$

Therefore, $\phi^{(n)}(t)$ is a unique global weak solution of (81). We can verify the existence of random dynamical systems generated by the global weak solution of (81).

\section{Acknowledgments}

The authors would like to thank an anonymous referee for valuable comments. Supported by the NSF of China (no. 10971225, no. 11028102, no. 11271013, and no. 91130003), the SRF for ROCS, SEM, and the NSF Grant 1025422.

\section{References}

[1] D. Applebaum, Lévy Processes and Stochastic Calculus, vol. 116 of Cambridge Studies in Advanced Mathematics, Cambridge University Press, Cambridge, UK, 2nd edition, 2009.

[2] S. Peszat and J. Zabczyk, Stochastic Partial Differential Equations with Lévy Noise, vol. 113 of Encyclopedia of Mathematics and Its Applications, Cambridge University Press, Cambridge, UK, 2007.

[3] P. Constantin and C. Foias, Navier-Stokes Equations, Chicago Lectures in Mathematics, University of Chicago Press, Chicago, Ill, USA, 1988.

[4] B. Ferrario, "The Bénard problem with random perturbations: dissipativity and invariant measures," Nonlinear Differential Equations and Applications, vol. 4, no. 1, pp. 101-121, 1997.
[5] C. Foias, O. Manley, and R. Temam, "Attractors for the Bénard problem: existence and physical bounds on their fractal dimension," Nonlinear Analysis: Theory, Methods \& Applications, vol. 11, no. 8, pp. 939-967, 1987.

[6] J. Lee and M.-Y. Wu, "Ergodicity for the dissipative Boussinesq equations with random forcing," Journal of Statistical Physics, vol. 117, no. 5-6, pp. 929-973, 2004.

[7] P. Brune, J. Duan, and B. Schmalfuß, "Random dynamics of the Boussinesq system with dynamical boundary conditions," Stochastic Analysis and Applications, vol. 27, no. 5, pp. 1096-1116, 2009.

[8] J. Duan, H. Gao, and B. Schmalfuß, "Stochastic dynamics of a coupled atmosphere-ocean model," Stochastics and Dynamics, vol. 2, no. 3, pp. 357-380, 2002, Special issue on stochastic climate models.

[9] J. Duan and A. Millet, "Large deviations for the Boussinesq equations under random influences," Stochastic Processes and Their Applications, vol. 119, no. 6, pp. 2052-2081, 2009.

[10] C. Sun, H. Gao, J. Duan, and B. Schmalfuß, "Rare events in the Boussinesq system with fluctuating dynamical boundary conditions," Journal of Differential Equations, vol. 248, no. 6, pp. 12691296, 2010.

[11] Z. Brzezniak and J. Zabczyk, "Regularity of Ornstein-Uhlenbeck processes driven by a Lévy white noise," Potential Analysis, vol. 32, no. 2, pp. 153-188, 2010.

[12] J. Lions, E. Magenes, and P. Kenneth, Non-Homogeneous Boundary Value Problems and Applications, Springer, New York, NY, USA, 1972.

[13] Z. Brzezniak, "Asymptotic compactness and absorbing sets for stochastic Burgers' equations driven by space-time white noise and for some two-dimensional stochastic Navier-Stokes equations on certain unbounded domains," in Stochastic Partial Differential Equations and Applications, VII, vol. 245 of Lecture Notes in Pure and Applied Mathematics, pp. 35-52, Chapman \& Hall/CRC, Boca Raton, Fla, USA, 2006.

[14] Z. Brzezniak, "Stochastic partial differential equations in Mtype 2 Banach spaces," Potential Analysis, vol. 4, no. 1, pp. 1-45, 1995.

[15] Z. Brzezniak and Y. Li, "Asymptotic compactness and absorbing sets for $2 \mathrm{D}$ stochastic Navier-Stokes equations on some unbounded domains," Transactions of the American Mathematical Society, vol. 358, no. 12, pp. 5587-5629, 2006.

[16] J. Bertoin, Lévy Processes, vol. 121 of Cambridge Tracts in Mathematics, Cambridge University Press, Cambridge, UK, 1996.

[17] K. Ito and F. Kappel, Evolution Equations and Approximations, vol. 61 of Series on Advances in Mathematics for Applied Sciences, World Scientific, River Edge, NJ, USA, 2002.

[18] L. Arnold, Random Dynamical Systems, Springer Monographs in Mathematics, Springer, Berlin, Germany, 1998.

[19] S. S. Sritharan and P. Sundar, "Large deviations for the twodimensional Navier-Stokes equations with multiplicative noise," Stochastic Processes and Their Applications, vol. 116, no. 11, pp. 1636-1659, 2006. 


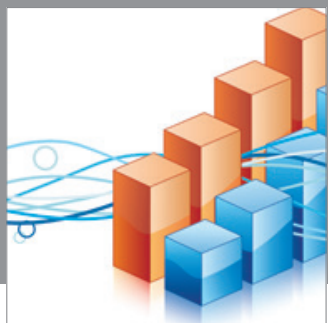

Advances in

Operations Research

mansans

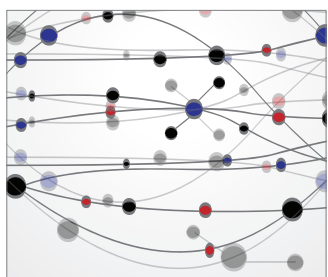

The Scientific World Journal
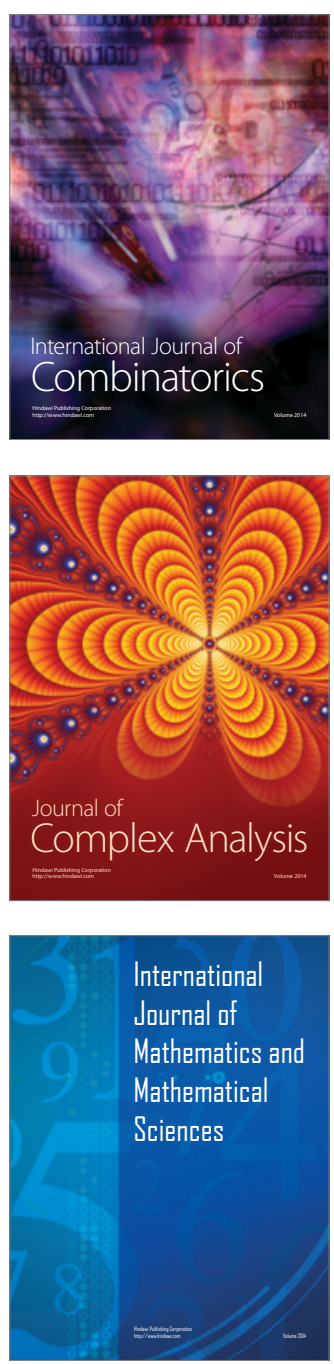
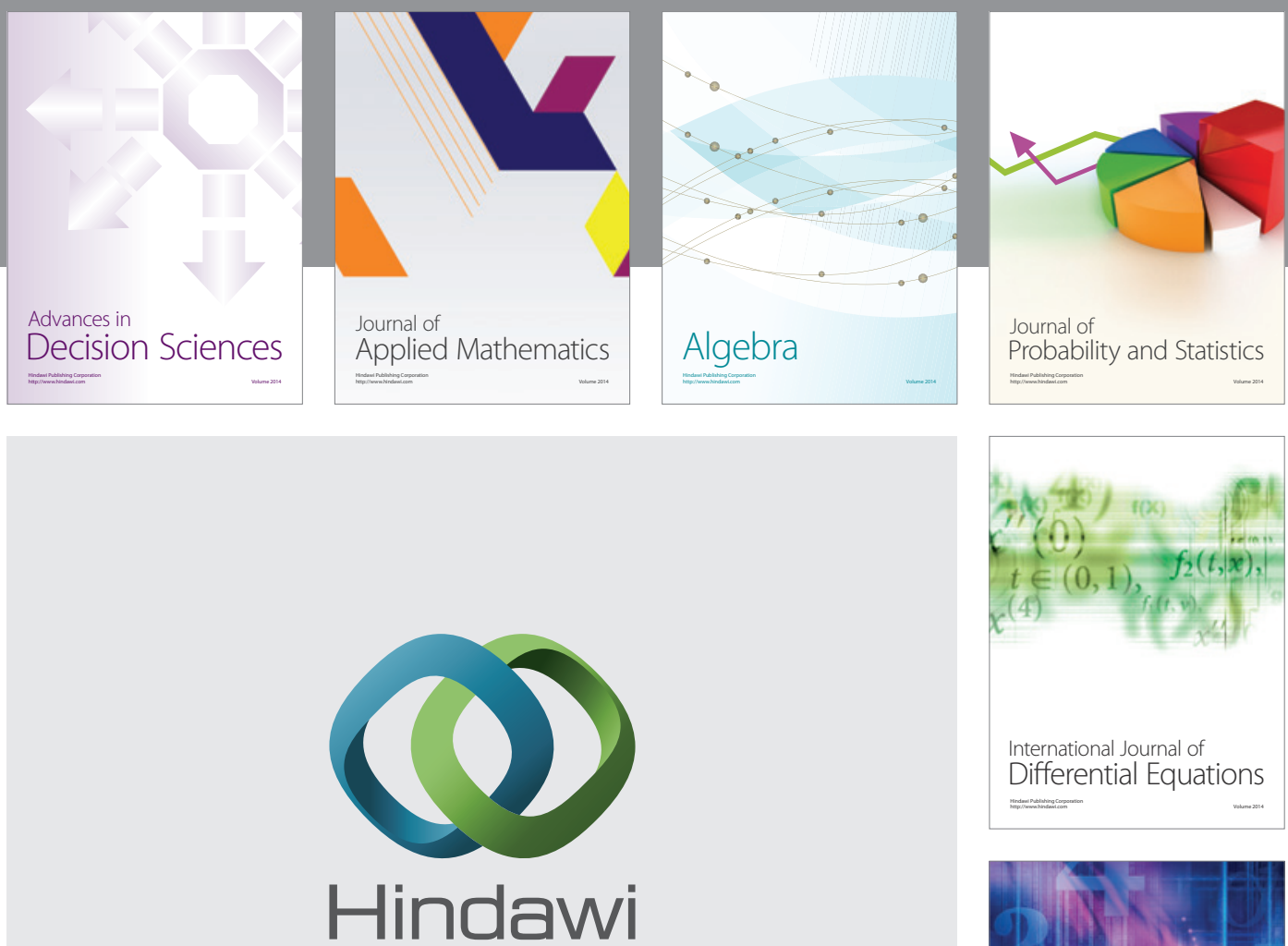

Submit your manuscripts at http://www.hindawi.com
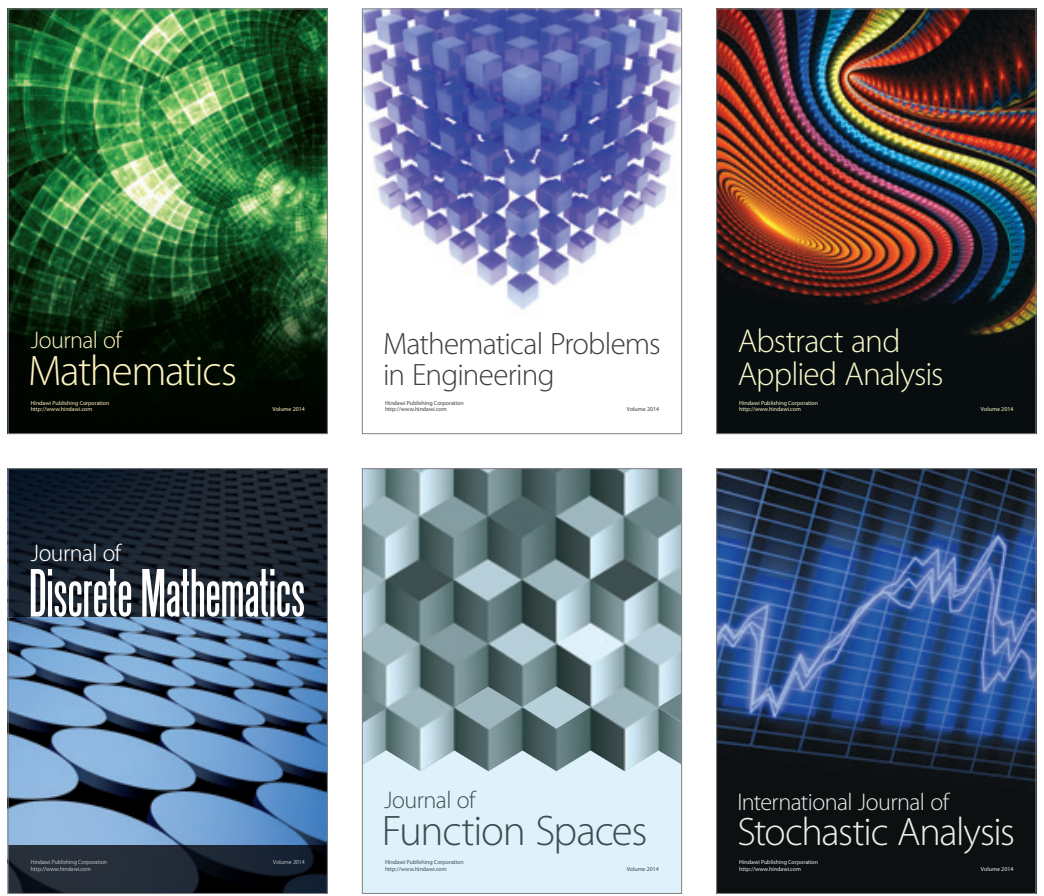

Journal of

Function Spaces

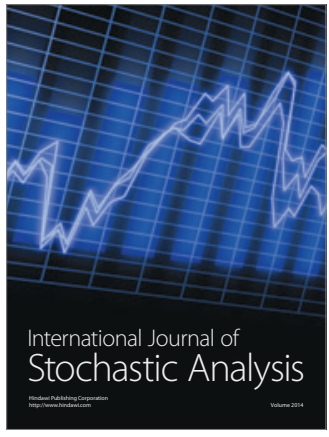

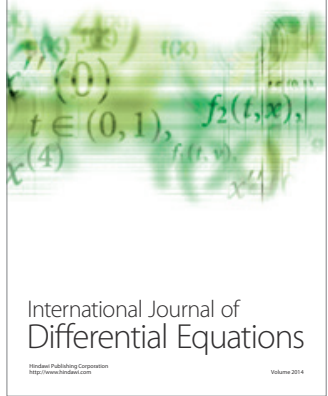
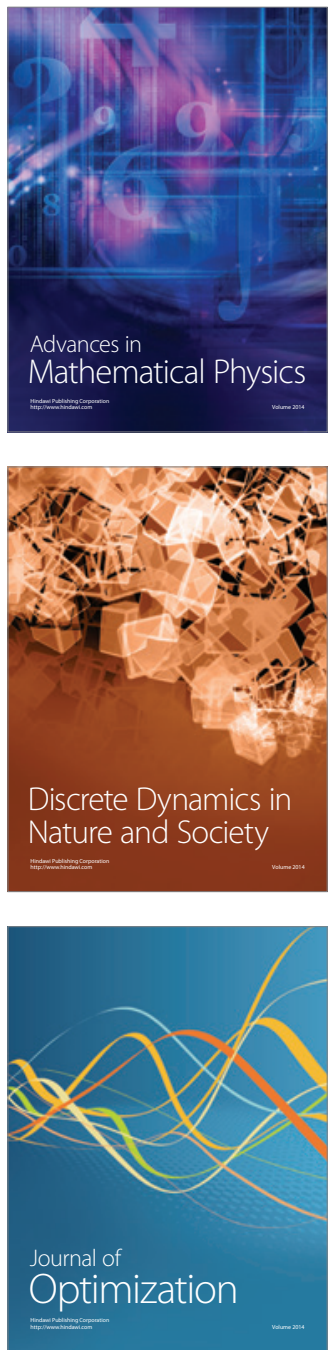